Ann. Biol. anim. Bioch. Biophys., 1979, 19 (3 B), 715-721.

\title{
Etude in vivo chez le rat de l'influence du glucose et du fructose sur le métabolisme intestinal et hépatique
}

\author{
par Elyett GUEUX, C. DEMIGNÉ *, C. RÉMÉSY* \\ Laboratoire d'Hydrologie et de Pharmacologie, Foculté de Médecine \\ Place Henri Dunant, 63000 Clermont-Ferrand \\ * Laboratoire des Maladies Métaboliques, I.N.R.A., \\ Theix, Saint-Genès-Champanelle, 63110 Beaumont.
}

Summary. Influence of glucose and fructose on intestinal and hepotic metabolism in the rat in vivo.

Intestinal and hepatic metabolism has been studied in rats fed 12-day diets comprising 74 p. 100 of either glucose or fructose. Food was available during $8 \mathrm{hrs}$, and tissue was sampled on anesthetized animals $6 \mathrm{hrs}$ after the beginning of food intake. In the digestive tract, fructose was poorly converted into glucose (less than 10 p. 100), and enterocyte lactate production was similar with both hexoses, representing less than 10 p. 100 of the absorbed carbohydrates. About 50 p. 100 of the absorbed glucose was taken up by the liver, as against more than 90 p. 100 of the fructose. Considering the transformation of fructose into glucose, the hepatic carbohydrate balance was very similar with the two diets in our sampling conditions. Decreased liver uptake of lactate accounted for the increased lactataemia with the fructose diet. This fact was possibly due to an increase of hepatic triose phosphate and pyruvate production, as fructose was mainly utilized in the fructose-1 phosphate pathway. Depletion of the hepatic ATP, induced by acute administration of fructose, was not observed in our adapted rats. As glycogen storage was more rapid with the fructose diet, triglyceride synthesis (an alternative pathway for the utilization of carbohydrates) certainly increased. This fact could account for the higher plasma triglycerides with the fructose diet, possibly associated with a lower response to the circulating insulin.

\section{Introduction.}

Le métabolisme du fructose a été largement étudié du fait que de nombreuses rations apportent des quantités importantes de ce glucide par l'intermédiaire du saccharose. D'autre part, les particularités métaboliques du fructose le distinguent nettement du glucose. Au niveau intestinal, le fructose est absorbé passivement ; toutefois, après activation dans l'entérocyle, il peut être isomérisé et transformé ainsi en glucose (Ginsburg et Hers, 1960). Cette transformation semble toutefois très faible chez l'homme

Adresse à laquelle doivent être envoyées les demandes de tirés à part : Laboratoire des Maladies Métaboliques, C.R.Z.V. Theix, 63110 Beaumont. 
et le rat (Herman, 1974). Une faible partie des hexoses absorbés est transformée en lactate, cette transformation seraif plus importante pour le fructose que pour le glucose dans la mesure où la phosphofructokinase ne serait plus une étape limitante (Srivastava ef Hübscher, 1966).

Le fructose absorbé est principalement métabolisé au niveau du foie où il provoque un certain nombre de modifications biochimiques étudiées en aigu (état de phosphorylation cellulaire, Woods, Eggleston et Krebs, 1970 ; Bode ef al., 1973), ou à plus long terme sur le métabolisme lipidique (Bruckdorfer, Khan et Yudkin, 1972 ; Griffaton et al., 1974).

Le but de cet article est de comparer le métabolisme intestinal en présence de glucose ou de fructose, ainsi que le métabolisme hépatique en reliant les modifications observées aux bilans des substrats éfudiés in vivo.

\section{Matériel et méthodes.}

Animaux et régimes. - Des rats mâles Sherman de $200 \mathrm{~g}$ ont reçu des régimes comprenant (en pourcentage) : glucose ou fructose 74 , caséine 15, huile d'arachide 5 , mélange minéral 5 et mélange vitaminique 1. Les mélanges minéraux et vitaminiques proviennent de UAR (Villemoisson/Orge). La nourriture était disponible de $9 \mathrm{~h}$ à $17 \mathrm{~h}$, avec une période obscure réglée de $9 \mathrm{~h}$ à $21 \mathrm{~h}$. Les consommations journalières (en matière sèche) étaient de 14,2 (régime glucose) et $14,3 \mathrm{~g} / \mathrm{j}$ (régime fructose) avec une croissance journalière d'environ $3 \mathrm{~g} / \mathrm{j}$ dans les 2 cas.

Prélèvements. - Après 12 jours d'adaptation au régime, les rats ont été anesthésiés au Nembutal $(40 \mathrm{mg} / \mathrm{kg}) 6 \mathrm{~h}$ après le début de la prise de nourriture ef environ $1 \mathrm{ml}$ de sang prélevé dans la veine hépatique, par ponction directe à l'intérieur du lobe gauche du foie, puis dans la veine porte et l'aorte. Le sang récolté sur héparine a été immédiatement centrifugé, une fraction du plasma a été congelée à $-20^{\circ} \mathrm{C}$ (dosage des acides gras libres et des triglycérides) et une autre fraction déprotéinisée par 2 volumes d' $\mathrm{HClO}_{4} 0,6 \mathrm{M}$. Les techniques de prélèvement hépatique ainsi que de dosage du glucose, glycogène, lactate, pyruvate, alanine et acides gras libres (AGL)

TABLEAU 1

Concentrations des méfabolites éfudiés ( $\mathrm{mM}$ ) dans le plasma artériel

\begin{tabular}{|c|c|c|}
\hline & Régime glucose & Régime fructose \\
\hline 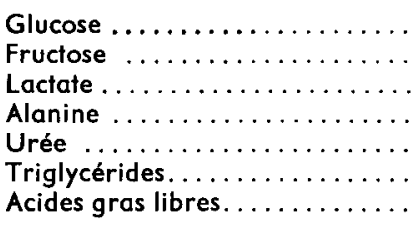 & $\begin{array}{l}8,26 \pm 0,52 \\
2,48 \pm 0,14 \\
0,59 \pm 0,03 \\
5,21 \pm 0,30 \\
0,98 \pm 0,07 \\
0,21 \pm 0,02\end{array}$ & $\begin{array}{l}7,51 \pm 0,22 \\
1,58 \pm 0,16 \\
3,37 \pm 0,18 * \\
0,69 \pm 0,05 \\
5,85 \pm 0,32 \\
1,60 \pm 0,17 * \\
0,34 \pm 0,04 *\end{array}$ \\
\hline
\end{tabular}

Les résultats sont les moyennes ( \pm SEM) pour 10 rats; * différences significatives entre les régimes $(P<0,05)$. 
ont été précédemment décrites (Rémésy, Demigné et Aufrère, 1978). Le dosage de I'ATP a été effectué selon la méthode de Lamprecht et Trautschold (1974), celui du fructose par la méthode à l'hexokinase après destruction du glucose préexistant par la glucose oxydase. Le dosage des triglycérides plasmatiques a été effectué par voie enzymatique, après hydrolyse directe dans le plasma puis déprotéinisation et mesure du glycérol, par la méthode de Wieland (1974). Les triglycérides hépatiques ont été déterminées par mesure du glycérol libéré après hydrolyse chimique (foie broyé dans la potasse alcoolique).

\section{Résultats et discussion.}

Les concentrations artérielles en métabolites sont présentées dans le tableau 1 : les glycémies ne sont pas significativement différentes entre les 2 régimes, par contre, du fructose est présent dans le sang artériel $(1,6 \mathrm{mM})$ avec le régime fructose. Ce régime élève significativement la concentration du lactate, des triglycérides et des AGL.

Les différences veine porte-artère (†abl. 2) mesurées en pleine phase absorptive

\section{TABLEAU 2}

Différences veine porte-artère $(\mathrm{mM})$ en glucose, fructose, lactate ef alanine

\begin{tabular}{lcc}
\hline & Régime glucose & Régime fructose \\
\hline Glucose $\ldots \ldots \ldots \ldots \ldots \ldots$ & $+6,10 \pm 0,43$ & $-0,05 \pm 0,10 *$ \\
Fructose $\ldots \ldots \ldots \ldots \ldots$ & $+6,00 \pm 0,50$ \\
Lactate $\ldots \ldots \ldots \ldots \ldots$ & $+0,87 \pm 0,16$ & $+0,98 \pm 0,20$ \\
Alanine $\ldots \ldots \ldots \ldots$ & $+0,30 \pm 0,03$ & $+0,36 \pm 0,04$ \\
\end{tabular}

Les? résultats sont les moyennes $( \pm S E M)$ pour 10 rats ; * différences significatives entre les régimes $(P<0,05)$.

(6 h après le début du repas) sont peu différentes pour le glucose et le fructose. La transformation du fructose en glucose est probablement faible (différence artérioveineuse nulle pour le glucose), mais la consommation permanente de glucose par le tube digestif pourrait masquer une faible formation de glucose (Topping et Mayes, 1971). Cette conversion est également faible chez l'homme, à la différence de certaines espèces (Heinz et al., 1975). Il n'y a pas de différence notable dans les libérations de lactate par le tube digestif avec les 2 régimes, et cette libération ne représente qu'une faible fraction de l'absorption nette du glucose ou du fructose (moins de 10 p. 100), ce qui est en contradiction avec les chiffres très élevés cités par Herman (1974) pour le fructose. II est possible toutefois que les potentialités de transformation du fructose en lactate s'expriment peu en raison des laciatémies élevées : en effet, Windmueller et Spaeth (1978) ont montré que la libération de lactate par le jéjunum diminuait avec l'élévation de la lactatémie. 
Les concentrations hépatiques en glycogène, lactate, pyruvate et triglycérides sont plus élevées avec le régime fructose (tabl. 3), alors que celles en glucose sont plus faibles. Les différences modérées dans les concentrations en pyruvate peuvent s'expliquer par la baisse de captation du lactate (cf. bilans hépatiques) bien que la production de trioses phosphates soit plus élevée avec le régime fructose. La forte activation du fructose par le foie ne s'accompagne pas d'une baisse de l'ATP. Par contre, une injection de fructose à des rats à jeun non adaptés $(2 \mathrm{~g} / \mathrm{kg}$ ) fait diminuer le taux d'ATP de 2,8 à $1,5 \mu \mathrm{mol} / \mathrm{g}$ de foie au bout de $30 \mathrm{mn}$. Ces résultats confirment les observations de Romsos ef Leveillé (1974) sur l'absence de diminution de l'ATP chez des animaux adaptés.

TABLEAU 3

Concentrations hépatiques en métabolites

\begin{tabular}{|c|c|c|}
\hline & Régime glucose & Régime fructose \\
\hline $\begin{array}{l}\text { Glycogène } \ldots \ldots \ldots \ldots \ldots \ldots \\
\text { Glucose } \ldots \ldots \ldots \ldots \ldots \ldots \ldots \\
\text { Lactate } \ldots \ldots \ldots \ldots \ldots \ldots \ldots \\
\text { Pyruvate } \ldots \ldots \ldots \ldots \ldots \ldots \\
\text { Triglycérides } \ldots \ldots \ldots \ldots \ldots \ldots \\
\text { ATP } \ldots \ldots \ldots \ldots \ldots \ldots\end{array}$ & $\begin{array}{l}62 \pm 4 \\
10,60 \pm 0,50 \\
3,50 \pm 0,30 \\
0,26 \pm 0,02 \\
10,2 \pm 0,8 \\
2,85 \pm 0,15\end{array}$ & $\begin{array}{l}92 \pm 6 * \\
8,61 \pm 0,60 * \\
4,50 \pm 0,50 * \\
0,36 \pm 0,02 * \\
18,4 \pm 2,4 * \\
2,72 \pm 0,08\end{array}$ \\
\hline
\end{tabular}

Les résultałs sont les moyennes $( \pm S E M)$ pour 10 rats, exprimés en $\mu \mathrm{mol} / \mathrm{g}$ de poids frais ; ${ }^{*}$ Différences significatives entre les régimes $(P<0,05)$.

TABLEAU 4

Bilans hépatiques $(\mathrm{mM})$ du glucose, fructose, lactate, alanine ef triglycérides

\begin{tabular}{|c|c|c|c|c|c|c|}
\hline & \multicolumn{3}{|c|}{ Régime glucose } & \multicolumn{3}{|c|}{ Régime fructose } \\
\hline & $\begin{array}{l}\text { Plasma } \\
\text { afférent }\end{array}$ & $\begin{array}{c}\text { Bilan } \\
\text { hépatique }\end{array}$ & $\begin{array}{l}\text { P. } 100 \\
\text { captation }\end{array}$ & $\begin{array}{l}\text { Plasma } \\
\text { afférent }\end{array}$ & $\begin{array}{c}\text { Bilan } \\
\text { hépatique }\end{array}$ & $\begin{array}{l}\text { p. } 100 \\
\text { captation }\end{array}$ \\
\hline $\begin{array}{l}\text { Glucose } \ldots \ldots \ldots \\
\text { Fructose } \ldots \ldots \ldots \\
\text { Lactate .... . . . } \\
\text { Alanine } \ldots \\
\text { Triglycérides . . . . }\end{array}$ & $\begin{array}{r}12,53 \pm 0,80 \\
= \\
3,05 \pm 0,25 \\
1,08 \pm 0,10 \\
1,08 \pm 0,11\end{array}$ & $\begin{array}{r}-2,17 \pm 0,25 \\
-1,13 \pm 0,14 \\
-0,28 \pm 0,05 \\
+0,04 \pm 0,01\end{array}$ & $\begin{array}{l}\frac{17}{37} \\
26\end{array}$ & $\begin{array}{l}7,47 \pm 0,50 * \\
5,78 \pm 0,75 \\
4,15 \pm 0,30 * \\
0,90 \pm 0,08 \\
1,61 \pm 0,11 *\end{array}$ & $\begin{array}{l}+1,12 \pm 0,30 * \\
-4,05 \pm 0,35 \\
-0,54 \pm 0,10 * \\
-0,19 \pm 0,04 \\
+0,15 \pm 0,02 *\end{array}$ & $\begin{array}{l}-70 \\
19 \\
21\end{array}$ \\
\hline
\end{tabular}

Les résultats sont les moyennes $( \pm S E M)$ pour 10 rats ; * différences significatives entre les deux régimes $(P<0,05)$.

Il a été admis que le débit hépatique se répartissait à raison de 70 p. 100 pour la veine porte et 30 p. 100 pour l'artère hépatique (Greenway et Stark, 1971) en considérant que le sang artériel hépatique a une composition identique à celui du sang artériel dans l'aorte. Le sang afférent est donc : 0,7 (veine porte) $+0,3$ (artère). Le bilan hépatique est la différence entre les concentrations du plasma de la veine hépatique et du plasma afférent. Les bilans hépatiques positifs correspondent à une libération par le foie et les bilans négatifs à une captation. Le pourcentage de captation est le rapport du bilan hépatique sur le plasma afférent $\times 100$. 
Les données du tableau 4 ont été calculées à partir des concentrations plasmatiques dans les différents vaisseaux sanguins en considérant que le débit de l'artère hépatique représente environ 30 p. 100 du débit hépatique (Greenway et Stark, 1971). La captation du fructose apparaît nettement plus élevée que celle du glucose $(4,05$ contre $2,17 \mathrm{mM}$, soit 70 contre 17 p. 100). Une partie du fructose capté est libéré sous forme de glucose (environ 30 p. 100), si bien que le bilan hépatique en hexoses est peu différent avec les 2 régimes. On peut calculer sur les mêmes bases de débit que plus de 90 p. 100 du fructose absorbé est capté par le foie, contre environ la moitié pour le glucose. Ceci indique que les tissus périphériques disposent de relativement moins de glucose avec le régime fructose. La captation du lactate est plus faible avec le régime fructose, en accord avec les concentrations hépatiques plus élevées (ce point avait été suggéré par Topping et Mayes, 1976) et par conséquent la disponibilité en lactate pour les tissus périphériques sera plus élevée. Par contre, ces différences de captation hépatique ne se retrouvent pas pour l'alanine.

La libération hépatique des triglycérides est nettement plus élevée avec le régime fructose, ceci, alors que les bilans hépatiques en AGL sont pratiquement nuls pour les 2 régimes (résultats non présentés). En supposant que ces triglycérides sont surtout synthétisés à partir des hexoses captés, la libération des triglycérides par le foie pourrait rendre compte de l'utilisation d'environ $2 \mathrm{mM}$ de fructose ou de $0,5 \mathrm{mM}$ de glucose, soit près de 50 p. 100 du fructose et 25 p. 100 du glucose captés.

L'activité des enzymes activant le fructose est certainement responsable des captations très élevées que nous enregistrons, de plus, le gradient plasma/foie est très favorable puisque chez nos animaux le fructose hépatique était extrêmement faible. Les activités de la fructokinase ef de la fructose-1P aldolase sont voisines et la triokinase n'est pas limitante chez le rat comme chez d'autres espèces (Heinz, 1972). Cependanf, ce n'est que par des injections en aigu de fructose qu'il a été démontré qu'une baisse de I'ATP et du $P_{i}$ entraîne une activation de l'AMP déaminase conduisant à l'IMP (Bode et al., 1973). Ce composé peut inhiber la fructose-1P aldolase et provoquer ainsi une accumulation de fructose-1P (Woods, 1972). Toutefois, l'adaptation de la production d'ATP n'est peut-être pas totale en début de période absorptive où l'on assiste à une élévation plus rapide du glycogène hépatique avec des régimes riches en fructose (résultats non présentés). En effet, la chute du $P_{i}$ peut correspondre à une activation de la glycogène synthétase, comme l'élévation du fructose-1P à l'inhibition de la phosphorylase (Hue, 1974).

II est vraisemblable que la production de pyruvate est fortement accrue avec les régimes fructose que ce soit par l'absence de contrôle par la phosphofructokinase ou par l'activation de la pyruvate kinase par le fructose-1P (Eggleston et Woods, 1970). D'un autre côté, l'activité de la fructose-1,6-diphosphatase pourrait être augmentée par certains effecteurs tels que le 3-phosphoglycérate et le citrate. Le fructose permet donc le fonctionnement simultané de deux voies métaboliques généralement antagonistes : la néoglucogenèse et la glycolyse. L'équilibre entre ces deux voies dépend certainement d'un contrôle complexe, notamment endocrinien, ainsi que des réserves en glycogène dont la constitution semble relativement prioritaire. Lorsque le glycogène est rendu à un niveau élevé, il semble que la transformation du fructose en glucose $(30 \mathrm{p}$. 100 dans nos expériences) soit limitée par la glucose-6-phosphatase bien que son activité soit augmentée par les régimes fructose (Fitch et Chaikoff, 1960 ; Cohen, Briller 
et Shafrir, 1972). L'utilisation des chaînons carbonés vers le pyruvate et la lipogenèse peut ainsi devenir très importante, et de fait, il est admis que le fructose élève la lipogenèse hépatique (Bruckdorfer, Khan et Yudkin, 1972) malgré une réduction de l'insuline circulante selon ces auteurs. Ce fait pourrait favoriser une accumulation hépatique des triglycérides d'autant plus que la disponibilité en glycéro-P et la réestérification sont plus élevées (Topping et Mayes, 1976). Chez nos animaux, l'élévation des triglycérides plasmatiques avec le régime fructose provient certainement d'une libération par le foie plus élevée, mais il esi envisageable qu'une moindre insulinosécrétion ou une éventuelle résistance des tissus périphériques à l'insuline puisse intervenir (Reiser et Hallfrisch, 1977 ; Vrana, Fabry et Kazdova, 1978). Cette situation endocrinienne pourrait rendre compte de l'élévation des AGL plasmatiques.

L'adaptation à des régimes riches en fructose se situe donc principalement au niveau du foie qui devient le principal site d'utilisation des glucides de la ration, avec synthèse accrue des réserves à la fois glucidiques et lipidiques. Cette adaptation passe notamment par le maintien de l'ATP hépatique par des mécanismes à préciser, comme pour le problème de l'insulinosécrétion et de la sensibilité des tissus à l'insuline.

Commission CNERNA Digestion-Absorption/Association des Physiologistes. Paris 5-6 octobre 1978.

\section{Références}

BODE J. C., ZELDER O., RUMPELT H. J., WITTKAMP U., 1973. Depletion of liver adenosine phosphates and metabolic effects of intravenous infusion of fructose or sorbitol in man and in the rat. Europ. J. clin. Invest., 3, 436-441.

BRUCKDORFER K. R., KHAN I. H., YUDKIN J., 1972. Fatty acid synthetase activity in the liver and adipose tissue of rats fed with various carbohydrates. Biochem. J., 129, 439-446.

COHEN A. M., BRILLER S., SHAFRIR S., 1972. Effect of long-term sucrose feeding on the activity of some enzyme regulating glycolysis, lipogenesis and gluconeogenesis in rat liver and adipose tissue. Biochim. biophys. Acta, 279, 129-138.

EGGLESTON L. V., WOODS H. F., 1970. Activation of liver pyruvate kinase by fructose 1-phosphate. FEBS Lefters, 6, 43-45.

FITCH W. M., CHAIKOFF I. L., 1960. Extent and pattern of adaptation of enzymes activities in livers of normal rats fed diets high in glucose and fructose. J. biol. Chem., 235, 554-557.

GINSBURG V., HERS H. G., 1960 . On the conversion of fructose to glucose by guinea pig intestine. Biochim. biophys. Acta, 38, 427-434.

GREENWAY C. V., STARK R. D., 1971. Hepatic vascular bed. Physiol. Rev., 51, 23-65.

GRIFFATON G., ROZEN R., ARDOUIN B., LOWY R., 1974. Effects of lipogenic precursors in rat liver, according to the nature of the dietary carbohydrate. Enzyme, 17, 319-332.

HEINZ F., 1972. Metabolism of fructose in liver. Acta med. scand., 542, suppl., 27-36.

HEINZ F., SCHLEGEL F., KRAUSE P. H., 1975. Enzymes of fructose metabolism in human small intestine mucosa. Enzyme, 19, 93-101.

HERMAN R. H., 1974. Hydrolysis and absorption of carbohydrates, and adaptative responses of the jejunum, 146-172. In SIPPLE H. L., Mc NUTT K. W., Sugars in Nutrition. Acad. Press, London.

HUE L., 1974. The metabolism and toxic effects of fructose, 367-368. In SIPPLE H. L., MC NUTT K. W., Sugars in Nutrition. Acad. Press, London.

LAMPRECHT W., TRAUTSHOLD I., 1974. Adenosine-5'-triphosphate. Defermination with hexokinase and glucose-6-phosphate dehydrogenases, 2101-2110. In BERGMEYER H. U., Methods of enzymatic analysis. Verlag Chemie, Weinheim, R. F. A.

REISER S., HALLFRISCH J., 1977. Insulin sensivity and adipose tissue weight of rats fed starch or sucrose diets ad libitum or in meals. J. Nutr., 107, 147-155. 
RÉMÉSY C., DEMIGNÉ C., AUFRÈRE J., 1978. Inter-organ relationships between glucose, lactate and amino acids in rats fed on high-carbohydrate or high-protein diets. Biochem. J., 170, 321-329.

ROMSOS D. R., LEVEILLÉ G. A., 1974. Effect of diefary fructose on in vitro and in vivo fatty acid synthesis in the rat. Biochim. biophys. Acta, 360, 1-11.

SRIVASTAVA L. M., HÜBSCHER G., 1966. Glucose metabolism in the mucosa of the small intestine. Biochem. J., 100, 458-466.

TOPPING D. L., MAYES P. A., 1971. The concentrations of fructose, glucose and lactate in the splanchnic blood vessels of rats absorbing fructose. Nufr. Metabol., 13, 331-338.

TOPPING D. L., MAYES P. A., 1976. Comparative effects of fructose and glucose on the lipid and carbohydrate metabolism of perfused rat liver. Br. J. Nutr., 36, 113-126.

VRANA A., FABRY P., KAZDOVA L., 1978. Liver glycogen synthesis and glucose tolerance in rats adaptated to diets with a high proportion of fructose or glucose. Nufr. Metab., 22, 262-268.

WIELAND O., 1974. Glycerol. UV-Method, 1404-1409. In BERGMEYER H. U., Methods of enzymatic analysis. Verlag Chemie, Weinheim, R. F. A.

WINDMUELLER H. G., SPAETH A. E., 1978. Identification of ketone bodies and glutamine as the major respiratory fuels in vivo for postabsorptive rat small intestine. J. biol. Chem., 253, 69-76.

WOODS H. F., 1972. Hepatic accumulation of metabolites after fructose loading. Acta med. scand. 542, suppl., 87-103.

WOODS H. F., EGGLESTON L. K., KREBS H. A., 1970. The cause of hepatic accumulation of fructose 1 phosphate on fructose loading. Biochem. J., 119, 501-510. 